\title{
Tinnitus after head injury: evidence from otoacoustic emissions
}

\author{
Borka J Ceranic, Deepak K Prasher, Ewa Raglan, Linda M Luxon
}

\begin{abstract}
Objective-Tinnitus may be caused by a lesion or dysfunction at any level of the auditory system. This study explores cochlear mechanics using otoacoustic emissions in patients with tinnitus after head injury, in whom there seems to be evidence to support dysfunction within the CNS.

Methods-The study included 20 patients with tinnitus and other auditory symptoms, such as hyperacusis and difficulty in listening in background noise, after head injury, in the presence of an "intact" auditory periphery (normal or near normal audiometric thresholds). They were compared with 20 normal subjects and 12 subjects with head injury, but without tinnitus, who had similar audiometric thresholds. In all subjects otoacoustic emissions, including transient clickevoked (TEOAEs) and spontaneous otoacoustic emissions (SOAEs), were recorded, and a test of efferent medial olivocochlear suppression, consisting of recording of TEOAEs under contralateral stimulation, was performed.
\end{abstract}

Results-A significantly higher prevalence of SOAEs $(100 \%)$, higher TEOAE response amplitudes, and reduced medial olivocochlear suppression in patients with tinnitus in comparison with subjects without tinnitus have been found.

Conclusion-These findings have been interpreted to be an extracochlear phenomenon, in which the reduction in central efferent suppression of cochlear mechanics, leading to an increase in cochlear amplifier gain, was subsequent to head injury. Auditory symptoms in these patients seemed to constitute the "disinhibition syndrome".

(F Neurol Neurosurg Psychiatry 1998;65:523-529)

Keywords: tinnitus; head injury; cochlear mechanics; efferent suppression

Tinnitus is an auditory perception that is not caused by externally applied stimulation. It is assumed that tinnitus is a consequence of altered neural activity and may result from a lesion or dysfunction at any level of the auditory system. ${ }^{12}$ Therefore, the source of "tinnitogenic" activity could be anywhere in the auditory system, although it is thought to be most often located in the auditory periphery. $^{34}$

In some patients with tinnitus, there is a and in revised form 26 February 1998

Accepted 12 March 1998 leading to the perception of tinnitus, results from a lesion within the CNS, and this presumption would be supported by the finding of "intact" peripheral auditory structures. Such patients may be found among those whose tinnitus occurred after head injury, and their evaluation, using otoacoustic emissions, is the objective of this study.

There is a paucity of literature concerning tinnitus induced by head injury, ${ }^{56}$ and tinnitus in these cases is probably largely unreported. According to Vernon and Press, ${ }^{6} 8 \%$ of the patients from their tinnitus data registry $(n=1240)$ represent the group with head injury.

Otoacoustic emissions represent unique tools for examining the cochlea, and have revolutionised clinical audiology by allowing a direct communication with the sensory cells. Substantial evidence exists that otoacoustic emissions result from the non-linear motile characteristics of outer hair cells, through the action of the contractile elements, myosin and actin, contained in the infrastructure of outer hair cells, ${ }^{78}$ and, presumably, outer hair cell active motility is the basis for the high hearing sensitivity and frequency selectivity. As otoacoustic emissions are invariably associated with functioning outer hair cells, their presence is a reliable indicator of cochlear $(\mathrm{OHC})$ structural integrity, and their absence may indicate a cochlear lesion. Furthermore, by recording otoacoustic emissions, a subclinical cochlear lesion may be detected, as up to $30 \%$ of the OHC population may be damaged before any audiometric evidence in the quarter octaves pure tone audiometry from $0.125 \mathrm{kHz}$ to 16 kHz. ${ }^{9}$

However, besides being an expression of the cochlear structural status, otoacoustic emission may also give an indication of functional integrity of the mechanisms which control the cochlea. Cochlear activity is regulated or modulated by the CNS. ${ }^{10}$ The olivocochlear efferent system is a part of that regulatory complex, and its medial division, widely known as the medial olivocochlear system, seems to be of particular importance in the modulation of cochlear activity. The high density of the medial olivocochlear innervation, with about $95 \%$ of its fibres targeting the outer hair cells, and large direct synaptic contact with the soma of the outer hair cells, in comparison with the indirect innervation of the inner hair cells (IHCs), ${ }^{11}$ reflects the potential influence of this system on the control of cochlear mechanics.

The effect of the medial olivocochlear system on the cochlea can be assessed by contralateral acoustic stimulation, which presumably activates, predominantly crossed, fibres of the 
Table 1 Subjects included in the study

\begin{tabular}{lllll}
\hline & Subjects $(n)$ & Sex $(f / m)$ & Ears $(n)$ & Age (mean (SD), range) \\
\hline Patients with head injury and tinnitus & 20 & $12 / 8$ & 37 & $36(9,20-49)$ \\
Normal subjects & 20 & $12 / 8$ & 40 & $37(7,24-50)$ \\
Patients with head injury without tinnitus & 12 & $7 / 5$ & 23 & $34(8,21-50)$ \\
\hline
\end{tabular}

medial olivocochlear system bundle and alters cochlear mechanics, and thus otoacoustic emission.

Although the medial olivocochlear system has been the most studied part of the efferent system, its functional aspects are still not well understood. The system is classically considered to be inhibitory, ${ }^{12}$ the effect being shown in many studies as reduced otoacoustic emission response amplitude, obtained by presenting the stimuli against silent background, and simultaneous contralateral acoustic stimulation. ${ }^{10}{ }^{13}$ However, there is evidence to suggest that the medial olivocochlear system enhances transient stimuli if they are presented against a continuous background noise. ${ }^{14}$

The higher structures, above the superior olivary complex (medulla oblongata), can modulate the excitability of olivocochlear neurons - for example, inferior colliculi ${ }^{11}{ }^{15}$ - or cortical and subcortical pathways, ${ }^{16}$ and, indirectly, exert their influence on cochlear mechanics.

The suppressive effect of the medial olivocochlear system in tinnitus and other auditory dysfunction has already been investigated. Preliminary studies of recording otoacoustic emission under contralateral acoustic stimulation in patients with unilateral tinnitus (comparing findings in the ears with and without tinnitus in the same subject), have reported that contralateral acoustic stimulation is less effective in ears with tinnitus. ${ }^{17}$ However, subsequent studies $^{19-22}$ showed a significant intersubject variability and a diversity in results between studies, so that no general rule could have been drawn. In another study, ${ }^{23}$ medial olivocochlear system function was compared in subjects with noise induced hearing loss, with and without tinnitus, and with normal hearing, with and without tinnitus. Otoacoustic emission responses up to $1.5 \mathrm{kHz}$ only, due to the absent responses in higher frequency bands in noise induced hearing loss, were considered. A significant difference (an enhancement of otoacoustic emission in the presence of contralateral stimulation) was found and attributed to a global efferent dysfunction. By contrast, a study comparing the efferent effect between the normal hearing (pure tone audiometry (PTA), 125-8000 Hz) patients with tinnitus and normal control subjects, ${ }^{24}$ suggested "a likely impaired functioning of the medial olivocochlear system", with less pronounced medial olivocochlear system effect in patients with tinnitus.

Table 2 Patients according to the severity of head injury (n (\%))

\begin{tabular}{llll}
\hline & Minor & Moderate & Severe \\
\hline Patients with head injury and tinnitus & $6 / 20(30)$ & $11 / 20(55)$ & $3 / 20(15)$ \\
Patients with head injury without tinnitus & $2 / 12(16.7)$ & $8 / 12(66.6)$ & $2 / 12(16.7)$ \\
\hline
\end{tabular}

The diversity in these findings, some of them conflicting, is probably a result of different evaluation methods applied and the heterogeneity of tinnitus groups (for example, aetiological, audiometrical, or age related ).

In addition to tinnitus, the medial efferent effect seemed to be altered in hyperacusis, with an increase in emissions under contralateral stimulation reported in a single case, ${ }^{25}$ and reduced in obscure auditory dysfunction. ${ }^{26}$

\section{Materials and methods}

SUBJECTS

All subjects included in the study (table 1 ) had normal or nearly normal hearing, defined as hearing thresholds equal to or better than 25 $\mathrm{dB}$ HL at octave step frequencies from 0.25 to $4 \mathrm{kHz}$ and up to $30 \mathrm{~dB} \mathrm{HL}$ at $6-8 \mathrm{kHz}$, together with normal middle ear function, defined as the ear drum compliance from 0.3$1.7 \mathrm{~cm}^{3}$ and the peak middle ear pressure \pm 50 $\mathrm{daPa}$. This criterion was essential in view of the known influence of the transmission properties of the middle ear on otoacoustic emission. ${ }^{27}{ }^{28}$

\section{Patients with tinnitus after head injury}

Twenty consecutive patients attended a neurootology clinic, with tinnitus lasting at least 1 year after head injury due to a road traffic accident $(13 / 20,65 \%)$, sport injury $(3 / 20,15 \%)$, blow to the head $(2 / 20,10 \%)$, or a fall $(2 / 20$, $10 \%)$. According to the classification of head injuries for severity, ${ }^{29}{ }^{30}$ six (30\%) patients sustained minor (post-traumatic amnesia lasted less then 3 hours and no skull fracture), 11 $(55 \%)$ moderate (a skull fracture was identified, or post-traumatic amnesia lasted between 3 hours to 7 days), and three (15\%) severe head injury (post-traumatic amnesia lasted more than 7 days) (table 2).

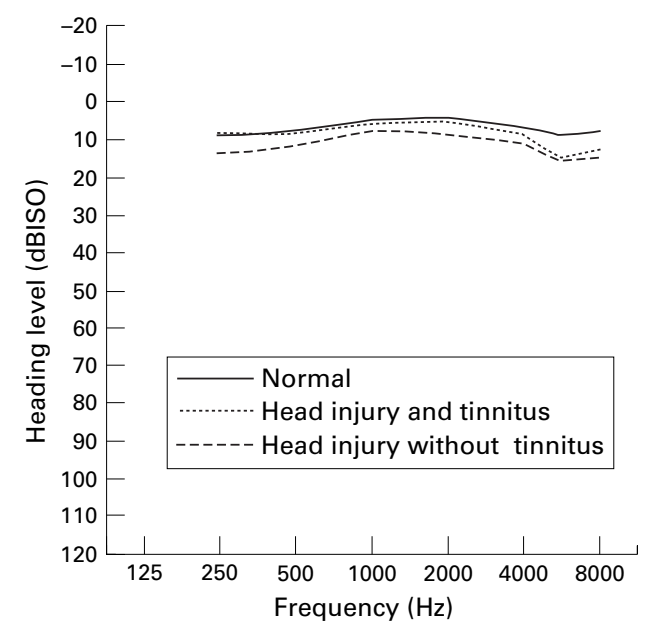

Figure 1 Mean pure tone audiometric thresholds for all groups. 
Table 3 Tympanometric measures (mean (SD)) in patients with tinnitus and in control groups

\begin{tabular}{llll}
\hline & $\begin{array}{l}\text { Eardrum } \\
\text { compliance }\left(\mathrm{cm}^{3}\right)\end{array}$ & $\begin{array}{l}\text { Peak pressure } \\
(\mathrm{dPa})\end{array}$ & $\begin{array}{l}\text { Ear canal } \\
\text { volume }\left(\mathrm{cm}^{3}\right)\end{array}$ \\
\hline Patients with head injury and tinnitus & $0.7(0.3)$ & $5.6(15)$ & $1.2(0.4)$ \\
Normal subjects & $0.8(0.3)$ & $-0.7(9)$ & $1.4(0.4)$ \\
Patients with head injury without tinnitus & $0.6(0.2)$ & $-0.4(9)$ & $1.3(0.4)$ \\
\hline
\end{tabular}

All patients complained of tinnitus of complex composition, which included different sounds, variable in pitch and volume and aggravated by environmental noise. Other auditory complaints included hyperacusis and difficulty in listening in background noise.

Apart from auditory disorders, other aspects of head injury in these patients have not been taken into consideration in this study.

\section{Patients with head injury, but without auditory complaints}

Twelve consecutive patients who sustained head injury, due to a road traffic accident (seven, 58.3\%), blow to the head (two, 16.7\%), sports injury (two, $16.7 \%$ ), or due to a fall (one, $8.3 \%$ ), were included in the study. Two of them $(16.7 \%)$ had minor, eight $(66.6 \%)$ had moderate, and two $(16.7 \%)$ had severe head injury.

Normal subjects without auditory complaints Twenty consecutive volunteers were selected to match the group with tinnitus for age and sex. This control group was introduced at a later stage of the study, after the finding of significantly reduced otoacoustic emission responses in the group without tinnitus in comparison with the group with tinnitus. The lower level of otoacoustic emission responses might result from a lower gain of the cochlear amplifier, but might also be a consequence of outer hair cell damage. As otoacoustic emissions represent the response based on the outer hair cell population, a scattered lesion of the outer hair cells could lead to a reduction in amplitude, without significant change in the microstructure of the response pattern. ${ }^{31}$

Therefore, by examining the spectral bands of the otoacoustic emission response, this type of outer hair cell lesion would not be identified.

The introduction of the group of normal subjects as another control group was considered to be adequate, as there is no reason to suspect that the group with tinnitus after head injury was not a part of the normal population before head injury.

PROTOCOL

All patients underwent a protocol which included an interview to obtain relevant information; otoscopy ( to exclude visual evidence of ear disease); standard pure tone audiometry;

Table 4 Mean (SD) TEOAE and noise levels in patients with tinnitus and control groups

\begin{tabular}{lll}
\hline & TEOAE responses (dB SPL) & Noise (dB SPL) \\
\hline Patients with head injury and tinnitus & $12.8(4)^{\star}$ & $-1.0(1)$ \\
Normal subjects & $8.0(3)$ & $-1.5(1)$ \\
Patients with head injury without tinnitus & $6.2(4)$ & $-0.9(1)$ \\
\hline${ }^{\star} \mathrm{p}<0.05$. & \\
TEOAE=transient click evoked otoacoustic emission.
\end{tabular}

tympanometry, and acoustic reflexes, the first of which was necessary to ascertain comparable middle ear properties between the groups; auditory brainstem responses to assess, together with acoustic reflex, the integrity of auditory pathways up to the superior olivary complex; recording of transient click evoked otoacoustic emissions (TEOAEs), spontaneous otoacoustic emissions (SOAEs); and an olivocochlear suppression test.

\section{OTOACOUSTIC EMISSIONS}

In this study TEOAEs and SOAEs were recorded using an Otodynamic ILO88/92 Analyser, version 3.4, in its default setting.

\section{TEOAE recording}

The stimulus presentation, data recording, averaging, and spectrum analysis have been carried out as described by Kemp et $a .^{32}$ Briefly, the stimuli were unfiltered rectangular clicks (bandwidth $5 \mathrm{kHz}$ ), duration of $80 \mu \mathrm{s}$, presented at repetition rate $50 / \mathrm{s}$, with the peak reception level at 80 (SD 3) dB sound pressure level (SPL). They were presented in non-linear mode (4 clicks with every fourth click of reversed polarity and $10 \mathrm{~dB}$ increase in amplitude), which cancels the linear portion of the stimulus and response, so that non-linear cochlear emissions can be extracted. The number of sweeps during the period of collection was 260 and the poststimulus analysis was $2.5-20 \mathrm{~ms}$.

The random noise contamination, the main sources of which are low frequency biological noise from the subject and ambient noise, was controlled by setting the rejection threshold at $47.3 \mathrm{~dB}$ SPL.

The following data, supplied by the ILO92 software, have been considered:

- The overall TEOAE response.

- The difference (A-B) of the two averaged waveforms, which is a good estimate of the noise level, to ensure comparable noise levels between the groups, preventing bias in TEOAE responses.

- The signal to noise $(\mathrm{S} / \mathrm{N})$ ratio in the bands (about $1 \mathrm{kHz}$ width) centred at 1, 2,3, 4, and $5 \mathrm{kHz}$, as the simplest form of TEOAE spectral analysis.

This analysis was performed to identify subclinical cochlear lesions (absence of the response in a spectral band) which may influence the TEOAE response.

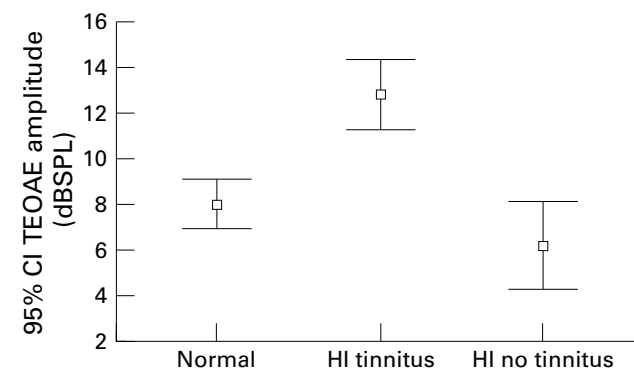

Figure 2 Means (95\% CIs) of overall TEOAE amplitude responses of subjects in normal and head injury (HI) groups. 

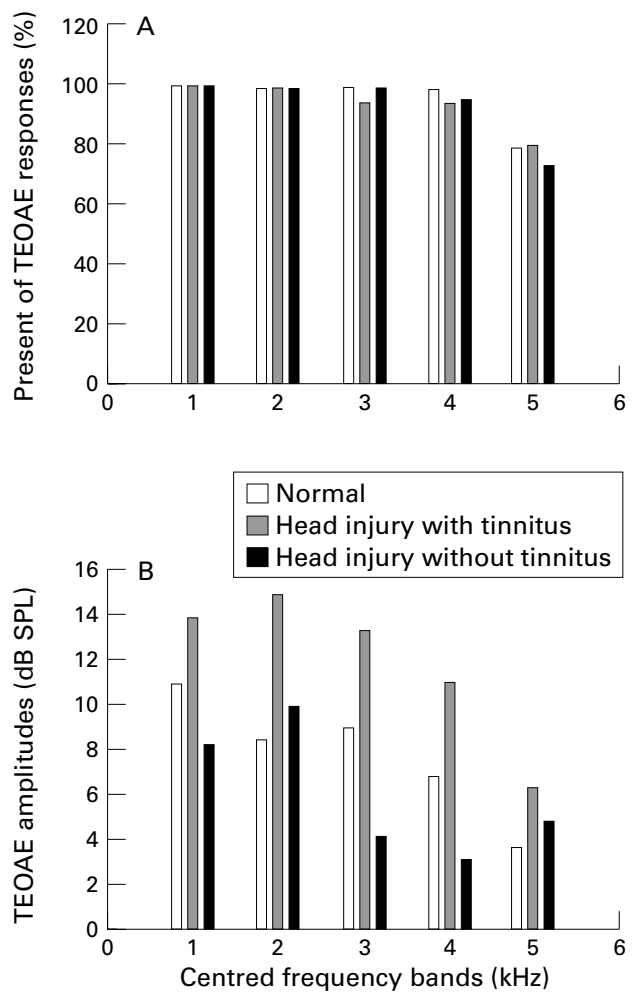

Figure 3 TEOAE spectral band analysis for subjects in normal and head injury groups. (A) Presence of TEOAE responses (\%). (B) Mean TEOAE amplitudes (dB SPL).

\section{$S O A E$ recording}

Synchronised SOAEs were recorded using a single $80 \mu \mathrm{s}$ click at around $75 \mathrm{~dB}$ SPL, presented in $80 \mathrm{~ms}$ intervals. As most of the subsequent response lasts less than $20 \mathrm{~ms}$, the microphone signal, averaged over a $20-80 \mathrm{~ms}$ poststimulus period, represents mostly spontaneous cochlear activity. Typically, 260 responses were averaged and Fast Fourier transformation (FFT) analysis was performed in the spectral band from 0 to $6250 \mathrm{~Hz}$, with a resolution of $12.3 \mathrm{~Hz}$. The presence of SOAEs was seen as spectral peaks of amplitude of at least 5 $\mathrm{dB}$ above the noise floor in the frequency range from 500 to $6250 \mathrm{~Hz}$. SOAEs at frequencies $<500 \mathrm{~Hz}$ were not considered due to the higher susceptibility to noise contamination in this frequency region.

Table 5 Results of SOAEs recorded in patients with tinnitus and in control groups

\begin{tabular}{|c|c|c|c|}
\hline & \multicolumn{2}{|c|}{ Prevalence (\%) } & \multirow{2}{*}{$\begin{array}{l}\text { Number of } \\
\text { peaks lears }\end{array}$} \\
\hline & lears & /subjects & \\
\hline Patients with head injury and tinnitus & $92^{\star}$ & $100^{\star}$ & $4.4^{\star}$ \\
\hline Normal subjects & 38 & 50 & 1.7 \\
\hline Patients with head injury without tinnitus & 17 & 17 & 0.25 \\
\hline
\end{tabular}

Table 6 Suppression of TEOAEs by contralateral noise

\begin{tabular}{lll}
\hline & $\begin{array}{l}\text { Suppression }>1 d B \\
\text { lears/subjects }(\%)\end{array}$ & $\begin{array}{l}\text { Mean }(S D, \text { range }) \\
(d B)\end{array}$ \\
\hline Patients with head injury and tinnitus & $57 / 35^{\star}$ & $1.2^{\star}\left(1,{ }^{\star} 0-3.5\right)$ \\
Normal subjects & 100 & $2.1(1,1-5.8)$ \\
Patients with head injury without tinnitus & 100 & $1.7(0.6,1.1-3.8)$ \\
\hline${ }^{\star} \mathrm{p}<0.05$. & \\
TEOAE=transient click-evoked otoacoustic emission. &
\end{tabular}

The prevalence of SOAEs and the number of SOAE peaks/ear were determined.

Medial olivocochlear suppression test

This test consists of recording of TEOAEs with and without contralateral stimulation and the difference in responses is considered, at least in part, to be the medial olivocochlear effect.

A dual channel otoacoustic emission analyser was used, one channel (A) for ipsilateral and the other (B) for contralateral acoustic stimulation. For ipsilateral stimulation, a linear click at 60 (SD $3 \mathrm{~dB}$ ) SPL intensity, and for the contralateral, broad band noise $(0.50-6 \mathrm{kHz})$ at $40 \mathrm{~dB}$ sensation level (SL), were used, applying an alternating technique, a "difference B on/off" mode, from the ILO92 software. This mode allows alternating recording of TEOAE responses with and without contralateral stimulation. A total of 600 sweeps were recorded, in 10 groups of 60 sweeps. The average responses were directly computed and the difference obtained by their subtraction represented the suppression effect.

All data were analysed using SPSS, including routine statistical procedures such as $\chi^{2}$, Leven's independent $t$ test, Mann-Whitney $U$ test, and one way analysis of variance (ANOVA), at the significance level of $\mathrm{p}<0.05$.

\section{Results}

PURE TONE AUDIOMETRY

Results of pure tone audiometry confirmed normal or near normal hearing in all subjects and there were no significant differences in the mean threshold levels between the groups (fig 1).

\section{TYMPANOMETRY}

Tympanometric measures in all subjects were within normal ranges and the middle ear pressure was comparable between the groups. The results are summarised in table 3.

\section{ACOUSTIC REFLEXES}

In all subjects, acoustic reflexes, ipsilateral and contralateral, were lower than $100 \mathrm{~dB}$ across at least three adjacent frequencies, with the exception of one patient with tinnitus, in whom reflexes were increased.

\section{AUDITORY BRAINSTEM EVOKED RESPONSES}

In all 12 patients with head injury without tinnitus, and in 16 out of 20 patients with head injury and tinnitus, auditory brainstem evoked responses were normal. In one patient with tinnitus-the same patient with increased acoustic reflex-auditory brainstem evoked responses were abnormal, with unilateral delay of wave V. In the remaining three patients, auditory brainstem evoked responses could not be obtained due to the patient's oversensitivity to the acoustic stimuli.

\section{OTOACOUSTIC EMISSION TESTS}

TEOAEs

The mean of overall TEOAE amplitudes in patients with tinnitus was significantly higher than in normal subjects, as well as in patients with head injury but without tinnitus. This is 
$34 \mathrm{y}$

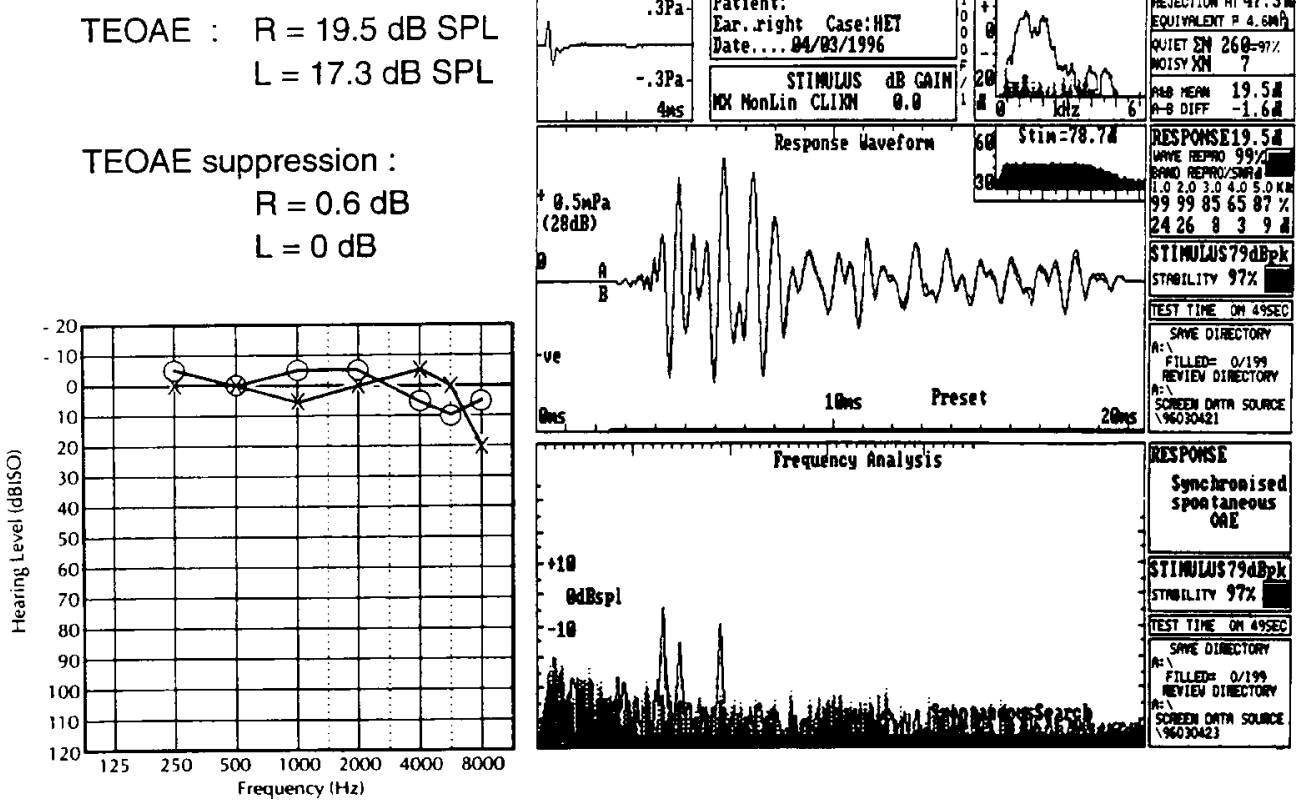

Figure 4 Findings in a patient with tinnitus, hyperacusis, and difficulty in listening in background noise, after severe head injury; only otoacoustic emission traces for the right ear are illustrated.

illustrated in fig 2, showing 95\% confidence intervals (95\% CIs) for the means for all three groups. This difference was seen in the presence of comparable noise level in all three groups (table 4).

TEOAE spectral analysis showed a similar distribution of the presence of TEOAE responses in $1,2,3,4$, and $5 \mathrm{kHz}$ bands (fig $3 \mathrm{~A}$ ). However, in patients with tinnitus, the mean TEOAE responses in all frequency bands were significantly higher than those in the control groups (fig $3 \mathrm{~B}$ ).

\section{SOAES}

The analysis of SOAE spectra showed a $100 \%$ prevalence of SOAEs in the group of 20 subjects with tinnitus. This was significantly higher than in the normal subjects $(10,50 \%)$ and the patients with head injury without tinnitus (two, 17\%). Similarly, the number of SOAE peaks were significantly higher in tinnitus (4.4/ear) than in the normal (1.7) and head injury without tinnitus (0.25) groups. The summarised results of SOAEs, including the prevalence of SOAE/ear, to indicate the presence of SOAEs in both ears, are shown in table 5 .

\section{Medial olivocochlear suppression test}

The suppression effect of the medial olivocochlear system was obtained by subtraction of the TEOAE responses under contralateral stimulation from those without contralateral stimulation. In all normal subjects this suppression effect was $\geqslant 1 \mathrm{~dB}$. Therefore, $1 \mathrm{~dB}$ was considered to be a cut off point, below which the medial olivocochlear suppression was labelled as reduced $(<1 \mathrm{~dB})$, or absent (0). Accordingly, all subjects in the control groups (20 normal subjects and 12 patients with head injury but without tinnitus) had normal efferent suppres- sion, whereas in patients with tinnitus, normal suppression was found in only seven out of 20 $(35 \%)$ subjects, or in the other words, in a significant number of subjects $(65 \%)$, medial olivocochlear suppression was found to be reduced or absent in one or both ears (table 6).

Figure 4 illustrates the findings in a patient with auditory complaints, including tinnitus, after severe head injury.

\section{Discussion}

Patients with tinnitus and other auditory complaints, including hyperacusis and difficulty in listening in background noise after head injury, showed significantly higher TEOAE amplitudes (12 (SD 4) dB SPL) than patients who had no auditory complaints, but who also sustained head injury (6.2 (SD 4) dB SPL). They were matched for sex and age, and had similar audiometric patterns. To exclude subclinical cochlear lesions, with the subsequent reduction of TEOAE amplitudes, and therefore to avoid potential bias in favour of the group with tinnitus, a simple spectral analysis, signal to noise ratio in 1, 2, 3, 4, and $5 \mathrm{kHz}$ centred bands, was performed. This analysis, which was considered to be, together with audiometric thresholds, an additional clinical criterion for normal hearing, did not show a significant difference in the distribution of the presence of TEOAE responses in frequency bands between the tinnitus and non-tinnitus head injury groups.

However, this procedure could not exclude scattered outer hair cell lesions, with a reduced number of outer hair cells contributing to the TEOAE response, leading to a reduction of amplitude, but without obvious change in the pattern of frequency dispersion.

Therefore, TEOAE responses, overall and in frequency bands, in patients with tinnitus after head injury were additionally compared with 
those in normal subjects who had neither tinnitus nor head injury. As expected, no subclinical cochlear lesions were detected in normal subjects, but TEOAE amplitude in patients with tinnitus still remained significantly higher than in this control group (8.0 (SD) $3 \mathrm{~dB}$ SPL).

A striking $100 \%$ prevalence of recordable SOAEs, as well as the largest number of SOAE spectral peaks/ear (4.4), in subjects with tinnitus was found, significantly higher than in both control groups; in normal subjects the prevalence of SOAE was 50\%, with 1.7 SOAE peaks/ear and in subjects with head injury without auditory complaints, SOAE prevalence was $17 \%$, with 0.25 SOAE peaks/ear. The SOAE prevalence in normal subjects was in agreement with previously reported results for such subjects. ${ }^{33}$

The magnitude of relative TEOAE reduction under contralateral acoustic stimulation, in comparison with TEOAE amplitude without contralateral stimulation, was found to $\mathrm{be} \geqslant 1 \mathrm{~dB}$ in all control subjects and these subjects were considered to have normal medial olivocochlear suppression (in this study $1 \mathrm{~dB}$ TEOAE reduction was a cut off point to separate subjects with normal and abnormal medial olivocochlear suppression). By contrast, in a significant number of patients with tinnitus $(65 \%)$, medial olivocochlear suppression was reduced $(<1 \mathrm{~dB})$ or absent $(0 \mathrm{~dB})$.

The effect of a lesion in the CNS (for example, at the brainstem level) on medial olivocochlear function leading to the absence or reduction of medial olivocochlear suppression has already been documented. ${ }^{13}{ }^{14}$ Whether the lesion affects the afferent, or efferent, or both parts of the olivocochlear reflex, is open to question.

The integrity of the afferent input and access to the efferent pathways was judged on the basis of normal stapedial reflexes and auditory brainstem responses in all subjects with the exception of four patients with tinnitus, one of whom had abnormal acoustic reflexes, and in three in whom auditory brainstem responses were not recorded because they could not tolerate the necessary acoustic stimulation. As the acoustic reflex, auditory brainstem responses, and olivocochlear reflex share the same ascending pathways, it is assumed that reduced or absent suppression of TEOAEs in these patients results from dysfunction of descending auditory pathway. However, for abnormal acoustic reflex and auditory brainstem evoked responses, the dysfunction could be in either the ascending or descending pathways.

In this study there is an indication of auditory efferent dysfunction involving the medial olivocochlear system in $65 \%$ of patients with tinnitus. In the remaining $35 \%$ of the patients, who exhibited similar characteristics with respect to the auditory symptoms and otoacoustic emission findings, the suppression test showed TEOAE reduction values $>1 \mathrm{~dB}$, implying normal medial olivocochlear function. As this test activates the medial olivocochlear system, the functioning of the rest of the efferent system, above the superior olivary complex (medulla oblongata), remains obscure. In view of the known multisynaptic connections of the medial olivocochlear system, within the inferior colliculi and via the inferior colliculi to the auditory cortex, suggesting a descending trisynaptic pathway from the cortex to the cochlea, it could be speculated that higher auditory pathways may be implicated in a more global efferent dysfunction, with the above described consequences.

The presence of significantly higher TEOAE responses, high prevalence of SOAEs with many SOAE spectral peaks, and reduced or absent medial olivocochlear efferent suppression in patients with tinnitus was interpreted to be due to an increase in the cochlear amplifier gain secondary to the dysfunction in the efferent control of cochlear mechanics (disinhibition of suppressive effect), subsequent to head injury.

There have already been suggestions that the size of emissions is related to the status of the medial olivocochlear system, ${ }^{34}$ which may be abnormal structurally - that is, with a demonstrable morphological lesion-or functionally - for example, with an imbalance of central neurotransmitters. This was illustrated in the experiment by Salonna et $a l^{35}$ in which intravenous atropine (an antagonist of acetylcholine, the principle neurotransmitter of the medial olivocochlear system), in 10 healthy human subjects led to a marked increase in emissions.

In normal conditions the central auditory system exerts its effect on the cochlea through efferently induced mechanisms of electromechanical transduction (outer hair cell electromotility), ${ }^{8}$ thus extending the dynamic range of the cochlea. The resulting cochlear amplification or attenuation is presumably a basis for the high sensitivity and frequency selectivity. However, reduced or absent medial olivocochlear suppression, as has been shown in this study in patients with head injury, may lead to a reduction in the dynamic range of the cochlea, leading further to a reduced ability of fine tuning and to difficulty to extracting transient stimuli in background noise. This could be a possible explanation for the symptom of difficulty in listening in background noise.

At the same time, the loss of a damping effect (attenuation) on afferent cochlear activity, normally produced by stimulation of the medial olivocochlear bundle, and an increase in amplifier gain causes an increase in the cochlear partition displacement as a response to auditory stimuli. This could be responsible for abnormal sensitivity to ordinary environmental sounds (hyperacusis). This increased auditory gain may also result in abnormal neural excitation, abnormal central sound processing, and, consequently, tinnitus.

It is apparent that tinnitus and other auditory complaints-hyperacusis and difficulty in listening in background noiseattributed to head injury and associated with normal peripheral auditory function (normal or nearly normal audiometric thresholds) and undamped otoacoustic emission, robust TEOAEs, and almost invariably recordable 
SOAE - are the consequences of an extracochlear phenomenon and constitute a clinical presentation which may be termed "disinhibition syndrome", subsequent to central efferent auditory dysfunction.

To summarise, this study has shown the potential value of otoacoustic emission in the assessment of tinnitus and other auditory complaints, providing information on structural integrity of the cochlea. It also gives insight into the functioning of the central control mechanisms. This information may contribute to a better understanding of the origin and mechanisms underlying tinnitus resulting from a lesion or dysfunction in the CNS.

Additionally, the assessment of medial olivocochlear system by recording otoacoustic emission under contralateral acoustic stimulation in a suspected lesion of the CNS could contribute to neuro-otological topographic diagnostics.

1 Møller AR. Pathophysiology of tinnitus. Ann Otol Rhinol

Laryngol 1984;93:39-44.
2 Jastreboff PJ. Phantom auditory perception (tinnitus): Jastreboff PJ. Phantom auditory perception (tinnitus):
mechanisms of generation and perception. Neurosci Res

3 Tonndorf J. Acute cochlear disorders; the combination of hearing loss, recruitment, poor speech discrimination, and tinnitus. Ann Otol Rhinol Laryngol 1980;89:353-8.

$4 \mathrm{McFadden} \mathrm{D}$. Tinnitus facts, theories and treatments. Washington, DC: National Academy Press, 1982

5 Lackner JR. The auditory characteristics of tinnitus resulting from cerebral injury. Exp Neurol 1976;51:54-62.

6 Vernon JA, Press LS. Characteristics of tinnitus induced by head injury. Arch Otolaryngol Head Neck Surg 1994;120: 547-51.

7 Brownell WE, Bader CR, Bertrand D, et al. Evoked mechanical responses of isolated cochlear outer hair cells. Science 1985;227:194-6.

8 Zenner HP. Motile responses in outer hair cells. Hear Res 1986;22:83-90.

9 Bohne B, Clark WW. Growth of hearing loss and cochlear lesion with increasing duration of noise exposure. In: lesion with increasing duration of noise exposure. In: Hamernik RP, Henderson D, Salvi RJ, eds. New perspectives on noise-ind

10 Collet L, Kemp DT, Veuillet E, et al. Effect of contralateral auditory stimuli on active cochlear micromechanical properties in human subjects. Hear Res 1990;43:251-62.

11 Warr WB, Guinan JJ Jr. Efferent innervation of the organ of Corti: two separate systems. Brain Res 1978;173:152-5.

12 Wiederhold ML. Physiology of the olivocohlear system. In Altschuler R, Bobbin R, Hoffman D, eds. Neurobiology of hearing. The cochlea. New York: Raven Press, 1986:349-70.

13 Ryan S, Kemp DT, Hinchcliffe R. The influence of contralateral acoustic stimulation on click-evoked otoacoustic emissions in humans. Br $\mathcal{F}$ Audiol 1992;25:391-7.

14 Kawase B, Delgutte, Liberman MC. Antimasking effects of the olivocochlear reflex. II. Enhancement of auditory-nerve response to masked tones. F Neurophysiol 1993;70:2533-49.
15 Rajan R. Electrical stimulation of the inferior colliculus at low rates protects the cochlea from auditory desensitisation. Brain Res 1990;506:192-204.

16 Attias J, Urbach D, Gold S, et al. Auditory event related potentials in chronic tinnitus with noise induced hearing loss. Hear Res 1993;71:106-13.

17 Veuillet E, Collet L, Disant F, et al. Tinnitus and medial cochlear efferent system. In: Aran JM, Dauman R, eds. Tinnitus 91. Proceedings of IV International Tinnitus Seminar, Bordeaux. Amsterdam: Kugler 1992:205-9.

18 Chéry-Croze S, Collet L, Morgon A. Medial olivo-cochlear system and tinnitus. Acta Otolaryngol (Stochk) 1993;113: $285-90$.

19 Chéry-Croze S, Moulin A, Collet L, et al. Is the test of medial efferent system function a relevant investigation in tinnitus? Br 7 Audiol 1994;28:13-15.

20 Chéry-Croze S, Truy E, Morgon A. Contralateral suppression of of transiently evoked otoacoustic emissions in tinnitus. Br F Audiol 1994;28:255-66.

21 Graham RL, Hazell JW. Contralateral suppression of transient evoked otoacoustic emissions: intra-individual variability in tinnitus and normal subjects. $\mathrm{Br} \mathcal{F}$ Audiol 1994;29:235-45.

22 Lind O. Transient-evoked otoacoustic emissions and contralateral suppression in patients with unilateral tinnitus. Scand Audiol 1995;25:167-72.

23 Attias J, Bresloff I, Furman V. The influence of the efferent auditory system on otoacoustic emissions in noise induced tinnitus: clinical relevance. Acta Otolaryngol (Stockh) 1996; 116:534-9.

24 Duchamp C, Morgon A, Chéry-Croze S. Tinnitus sufferers without hearing loss. In: Reich GE, Vernon JA, eds. Proceedings of the V International Tinnitus Seminar 1995, Portland, Oregon, USA. Portland, Oregon, USA: American Tinnitus Association 1996:266-9.

25 Collet L, Veuillet E, Bene J, et al. The effects of contralateral white noise on click-evoked emissions in normal and sensorineural ears: towards an exploration of the medial olivocochlear system. Audiology 1992;31:1-7.

26 Zhao F, Meredith R, Stephens SDG. Transient evoked otoacoustic emissions with contralateral stimulation in King-Kopetzky syndrome. $\mathcal{F}$ Audiol Med 1996;6:36-44.

27 Kemp DT. Physiologically active cochlear micromechanisms-one source of tinnitus. In: Evered D, Lowrenson G, eds. Tinnitus. (CIBA Foundation Symposium 85). London: Pitman Books, 1981:54-81.

28 Hauser R, Probst R, Harris FP. Effect of atmospheric pressure on spontaneous, transiently evoked and distortion product otoacoustic emissions in normal human ears. Hear Res 1993;69:133-45.

29 Symonds C. Concussion and its sequelae. Lancet 1962;i: $1-5$.

30 Berman JM, Fredrickson JM. Vertigo after head injury-a five year follow-up. I Otolaryngol 1978;7:237-45.

$31 \mathrm{He} N$, Schmiedt RA. Fine structure of the $2 \mathrm{f}_{1}-2 \mathrm{f}_{2}$ acoustic distortion product: changes with primary level. 7 Acoust Soc Am 1993;94:2659-69.

32 Kemp DT, Ryan S, Bray D. A guide to the effective use of otoacoustic emissions. Ear Hear 1990;11:93-105.

33 Probst R, Lonsbury-Martin BL, Martin GK. A review of otoacoustic emissions. F Acoust Soc Am 1991;89:2027-67.

34 Berlin CI, Hood LJ, Cecola RP, et al. Does type I afferent neural dysfunction reveal itself through lack of efferent suppression? Hear Res 1993;65:40-50.

35 Salonna I, Bartoli A, Longo S. Evoked otoacoustic emissions: effects of atropine. Boll Soc Ital Biol Sper 1990;66:167-71. 